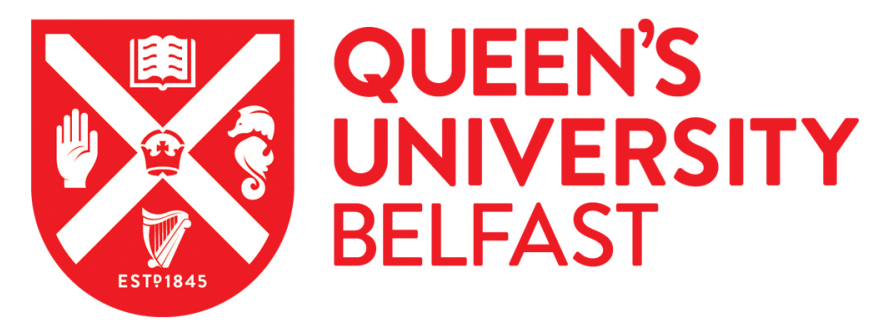

\title{
Optimisation of laser driven proton beams by an innovative target scheme
}

Ahmed, H., Kar, S., Cantono, G., Doria, D., Giesecke, A. L., Gwynne, D., Lewis, C. L. S., Macchi, A., Narsisyan, G., Naughton, K., Willi, O., \& Borghesi, M. (2017). Optimisation of laser driven proton beams by an innovative target scheme. Journal of Instrumentation, 12, [C06025]. https://doi.org/10.1088/1748-0221/12/06/C06025

\section{Published in:}

Journal of Instrumentation

\section{Document Version:}

Peer reviewed version

Queen's University Belfast - Research Portal:

Link to publication record in Queen's University Belfast Research Portal

\section{Publisher rights}

(c) 2017 IOP Publishing Ltd and Sissa Medialab srl. This work is made available online in accordance with the publisher's policies. Please refer to any applicable terms of use of the publisher.

\section{General rights}

Copyright for the publications made accessible via the Queen's University Belfast Research Portal is retained by the author(s) and / or other copyright owners and it is a condition of accessing these publications that users recognise and abide by the legal requirements associated with these rights.

Take down policy

The Research Portal is Queen's institutional repository that provides access to Queen's research output. Every effort has been made to ensure that content in the Research Portal does not infringe any person's rights, or applicable UK laws. If you discover content in the Research Portal that you believe breaches copyright or violates any law, please contact openaccess@qub.ac.uk. 


\title{
Optimisation of laser driven proton beams by an innovative target scheme
}

\author{
H. Ahmed ${ }^{a}$ S. Kar ${ }^{a, b, *}$ G. Cantono ${ }^{c}$ D. Doria ${ }^{a}$ A. L. Giesecke ${ }^{d}$ D. Gwynne ${ }^{a}$ C.L.S. Lewis ${ }^{a}$ \\ A. Macchi ${ }^{c}$ G. Narsisyan ${ }^{a}$ K. Naughton ${ }^{a}$ O. Willi ${ }^{d}$ M. Borghesi $^{a}$ \\ ${ }^{a}$ School of Mathematics and Physics, QueenâĂŹs University Belfast, Belfast BT7 INN, UK. \\ ${ }^{b}$ Central Laser Facility, Rutherford Appleton Laboratory, Didcot, Oxfordshire OX11 OQX, UK. \\ ${ }^{c}$ Department of Physics E. Fermi, Largo B. Pontecorvo 3, 56127 Pisa, Italy \\ ${ }^{d}$ Institut für Laser-und Plasmaphysik, Heinrich-Heine-Universität, Düsseldorf, Germany \\ E-mail: s.kar@qub.ac.uk
}

\begin{abstract}
Laser driven proton beams driven by the Target Normal Sheath Acceleration (TNSA) mechanism exhibit large divergence and a broad energy distribution with low particle number at high energy. Such undesirable characteristics of the beam can be controlled and optimised by employing a recently developed helical coil technique, which exploits the transient self-charging of solid targets irradiated by intense laser pulses. Highly chromatic focusing of the broadband proton beams was achieved by employing this technique at the TARANIS laser system, where the selected energy slice was tuned by varying the pitch of the coil. Using a longer coil of larger pitch, a quasi-collimated, narrow energy band proton beam of $\sim 10^{7}$ particles at $10 \mathrm{MeV}$ was achieved, through a combination of focussing, energy selection and in-situ post-acceleration. This technique may provide a platform for the next generation of compact, all-optical ion accelerators.
\end{abstract}

KEYwORDs: laser-driven, positive ions, accelerator, beam optics

${ }^{1}$ S. Kar (* Invited speaker at ELIMED 2016) 


\section{Contents}

1 Introduction 1

2 Physics underlying the HC scheme 2

3 Experimental arrangement $\quad 4$

4 Optimisation of TNSA proton beams using helical coil targets 5

4.1 Chromatic focusing and tunability 5

4.2 Post-acceleration by helical coil targets 5

5 Conclusions 6

\section{Introduction}

All-optical approaches to particle acceleration are currently attracting significant research interest internationally, in light of a wide range of potential applications including proton therapy [1], ion driven fast ignition (IFI) [2, 3], high energy density physics [4, 5], production of neutrons and radioactive isotopes $[6,7]$. Although characterised by exceptional transverse and longitudinal emittance, laser-driven ion beams (mainly driven by the target normal sheath acceleration mechanism (TNSA)[8]) currently have limitations in terms of the broad energy spectrum, large beam divergence and maximum ion energy, which constrains their applicative use and warrants further investigations aimed to the optimisation of the beam parameters [9]. Optimisation methods explored so far to are based on either by acting at the source, e.g by employing curved $[4,10]$ or microstructured targets, or on acting on the proton beam after the acceleration by employing micro-lenses[11], self-triggered electrostatic lenses [12,13], quadrupole magnets [14] and pulsed solenoids[15]). All these techniques allow a degree of control over the proton beams, but have limitations in terms of either the number of particles that can be delivered and/or requires propagation distances of $10 \mathrm{~s}$ of $\mathrm{cm}$, which stretches the selected bunch beyond the requirements of many of the applications.

In addition to addressing the divergence and broad spectrum of the beam, it is crucial, e.g. for medical applications, to find ways of increasing ion energy. Increasing the laser energy and intensity on target will naturally lead to an increase of the energy of TNSA-accelerated particles, although reaching 100s of $\mathrm{MeV}$ energies may require large, multi-PW systems [16]. Increasing particle flux and energy by secondary methods, such as coupling laser-driven ions to conventional RF stages for post-acceleration and beam control [17] is an approach currently being explored, which however, is arguably less attractive than an all-optical approach. A novel technique for simultaneous focussing, energy selection and post-acceleration has been recently developed [18], which exploits the transient self-charging of solid targets irradiated by intense laser pulses. In this scheme, a helical coil (HC) is attached to the rear surface of the interaction foil so that the protons 
generated from the rear surface of the foil propagate along the $\mathrm{HC}$ axis, while an electromagnetic (EM) pulse generated during the same interaction travels along the coil. For a given radius and pitch of the coil, it is possible to synchronise a section of the transiting proton spectrum with the longitudinal propagation of the electric field associated to EM pulse, which provides simultaneous focusing by its radial component and post-acceleration by its longitudinal component. As a proofof-principle, Kar. et al [18] demonstrated post-acceleration of the TNSA protons at a rate of $\sim 0.5 \mathrm{GeV} / \mathrm{m}$ inside a coil of $\sim 9 \mathrm{~mm}$ long (resulting in a net energy gain $\sim 5 \mathrm{MeV}$ ), with intrinsic beam collimation and energy selection, while employing HC targets at the 200 TW ARCTURUS laser system. In contrast to mechanisms such as TNSA, Radiation Pressure acceleration (RPA) [9], accelerating ions to high energies over a few microns using fields of the order of $\mathrm{TV} / \mathrm{m}$ and above, the HC target applies a more moderate field over an extended and controllable distance. In principle, this approach can be staged to increase ion energy sequentially in multiple targets driven by different laser pulses. It is to be noted that the acceleration gradient obtained at the ARCTURUS laser is already well beyond what can be achieved by conventional RF accelerator technologies. Demonstration of guided post-acceleration of ions at high energies ( $\sim 100 \mathrm{MeV}$ and beyond) by the $\mathrm{HC}$ technique, and developing approaches for operating the $\mathrm{HC}$ targets at a high repetition, would represent a significant development towards compact, cost-effective accelerators for a wide range of potential applications.

A detailed study of beam optimisation by this technique was carried out employing the $\mathrm{HC}$ targets at another university-based laser system, the TARANIS, which is hosted by Queen's university Belfast, UK. By varying the physical dimensions of the helical coil, experimental data demonstrate highly effective, and intrinsically chromatic proton beam guiding. As described in the ref [18], pronounced post-acceleration of protons can be achieved by using longer $\mathrm{HC}$ targets, mainly due to prolonged exposure of the electric field to the transiting protons. The data shows a highly collimated and quasi-monoenergetic ( $10 \%$ energy spread) proton beam, containing $>10^{7}$ particles at $10 \mathrm{MeV}$, achieved through a combination of focussing and post-acceleration of $\sim 7 \mathrm{MeV}$ protons, which are synchronised with the EM pulse. As the unsynchronized protons maintain their intrinsic beam divergence, the proton spectrum can be tailored, in principle, by deploying a spatial aperture at the beam output.

\section{Physics underlying the HC scheme}

The interaction of ultra-high intensity $\left(>10^{19} \mathrm{~W} \mathrm{~cm}^{-2}\right.$ ) lasers with solid targets produces a large population of relativistic electrons (energy of the order of $10 \mathrm{~s} \mathrm{MeV}$ ). Due to their high kinetic energy, a significant fraction of the electron population manages to escape from the target leaving the target positively charged. The loss of electrons is then compensated by a neutralising current from the ground, through the stalk(s)/wire(s) holding the target. Due to the extremely transient (ps) nature of the interaction, in a situation analogous to a charged capacitor being suddenly connected to a transmission line, the neutralising current effectively travels as an EM pulse along the wire connected to the target, highly localised in space, at any given point of time. The EM pulse produces a strong localised electric field in excess of $\mathrm{GV} / \mathrm{m}$ at the surface of the wire.

Information about the strength and nature of pulse propagation along a wire has been obtained experimentally by a self-proton probing technique [19], which has revealed a pulse duration of $\sim 20$ 

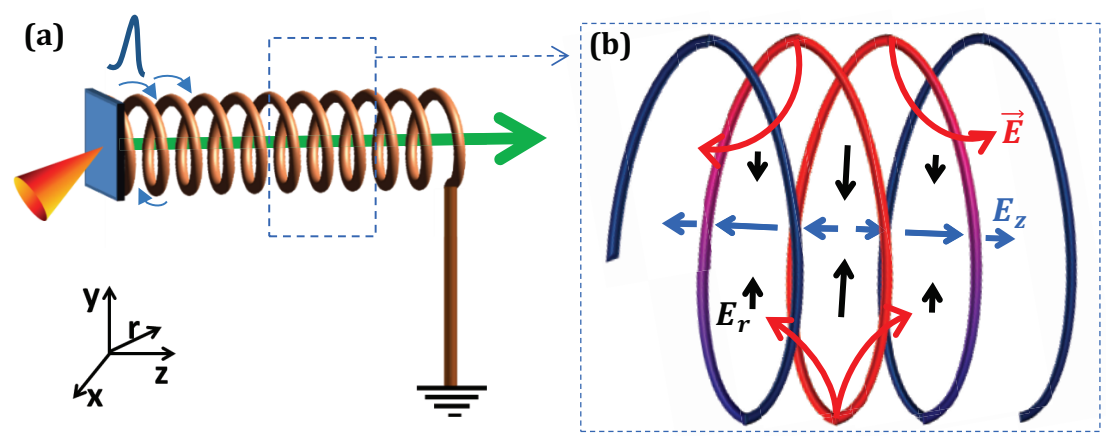

Figure 1. (a) Schematic of the HC target used for optimizing the beam parameters of laser-driven protons. The helical coil is attached to the laser-irradiated thin foil at one end and grounded at the other end. The EM pulse generated by the laser interaction travels around the proton-beam axis along the winding the helical coil. (b) Schematic showing the electric field configuration inside the coil at a given time. Assuming the red section of the coil representing the presence of the EM pulse at the given time, generating electric field lines represented by the red arrows, the black and blue arrows represent the radial $\left(E_{r}\right)$ and longitudinal $\left(E_{z}\right)$ components of the electric field, respectively, causing beam collimation and post-acceleration respectively. The length of the black and blue arrows represents the relative strength of the field at different locations with respect to the EM pulse. The figure is taken from the ref. [18].

ps FWHM travelling at a speed close to the speed of light [18-20], which corresponds to a length of $\sim 6 \mathrm{~mm}$ occupied by the pulse along a wire at a given time. The conceptual design of the HC target, as shown in the figure 1(a) was therefore developed by considering the following three key points - (1) use of thin wires optimises the surface charge density, hence the strength of the electric field around the wire; (2) helical winding of the wires allows a strong electric field region to exist inside the coil; (3) the single path for the EM pulse propagation set by the coil geometry allows synchronisation of the travelling electric field region within the coil with a narrow slice of the proton beam spectrum, which can be tuned by varying the coil diameter and pitch.

A schematic of the electric field configuration inside the coil at a given time is shown in figure 1(b). Assuming the red section of the coil represent the presence of the EM pulse at the given time, the radial $\left(E_{r}\right)$ and longitudinal $\left(E_{z}\right)$ components of the synchronised electric field act respectively towards focussing and acceleration of the transiting protons. Since the EM pulse travels with a constant speed ( $v_{\text {pulse }}$ ) close to the speed of light, the longitudinal speed of the travelling electric field region $\left(v_{\|}=p v_{\text {pulse }} / \sqrt{p^{2}+(2 \pi r)^{2}}\right.$, where, $\mathrm{r}$ and $\mathrm{p}$ are radius and pitch of the coil respectively) can be controlled by tuning the diameter and pitch of the coil, depending on the range of proton energies to be controlled by the HC target. The range of proton energies which remains synchronized with the EM pulse depends on their velocity dispersion, and therefore reduces gradually with the length of the coil. Within the synchronised bunch, the protons travelling slightly ahead with respect to the peak of the EM pulse will experience a longitudinal electric field in the forward direction. Using a simple charged-ring analogy [18], the peak electric field along the coil axis can expressed as $E_{z, \text { max }}=2 k Q / 3 \sqrt{3} r^{2}$, where $k$ is the Coulomb's constant and Q is the net charge contained within the pulse. A prolonged exposure to this field can produce an appreciable increase in proton energy, in a first approximation, equal to the $E_{z}$ multiplied by the 
length of the coil $(l)$ for instance. The protons in the trailing part of the synchronised bunch will experience a decelerating field and will lag behind the active field region after a small propagation distance. The deceleration of the slower protons is in fact favourable towards energy slicing and narrowing of the spectral peak at the coil output.

\section{Experimental arrangement}

The data presented in this paper were obtained from two experimental campaigns, under similar laser and target conditions, performed using the TARANIS laser [21] at Queen's University Belfast, employing the CPA pulse of duration $\sim 600 \mathrm{fs}$ with energy $\sim 5 \mathrm{~J}$ on the target. The short pulse was focused by a $\mathrm{f} / 3 \mathrm{off}$ axis parabola onto a Au foil of thickness approximately $10 \mu \mathrm{m}$, at an intensity $\sim 10^{19} \mathrm{~W} \mathrm{~cm}^{-2}$. The intensity contrast of the laser at $1-2$ ns before the main pulse was $\sim 10^{7}$. The targets were made by attaching a helical coil to the rear surface of the interaction foil, along the target normal direction, as shown in figure 1(b). Helical coils of various diameter, pitch and length were used to study the beam control and optimisation and were made of aluminium wire of diameter $80-100 \mu \mathrm{m}$.

A stack of multilayer radio chromic films (RCFs) was used in each shot to diagnose the spatial and spectral profile of the proton beam produced by the HC target. The RCF dose response was absolutely calibrated using batches of RCFs exposed to different known proton doses from a particle accelerator [22]. Due to the Bragg peak energy deposition profile of protons in matter, the proton dose deposited in a given layer of RCF is due primarily to a narrow range of proton energy, defined by the position of the RCF layer in the stack.

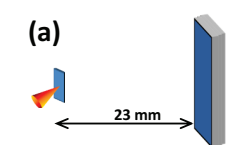

(c)

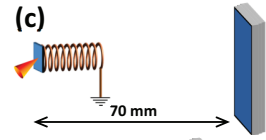

(e)

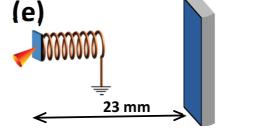

(b)

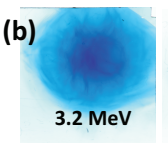

(d)

(f)

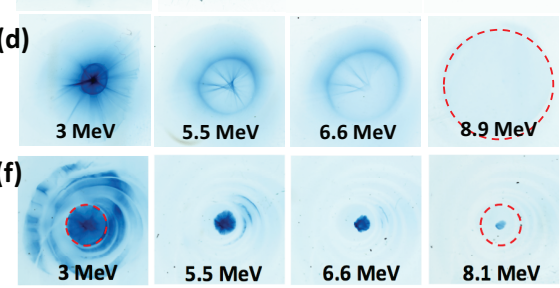

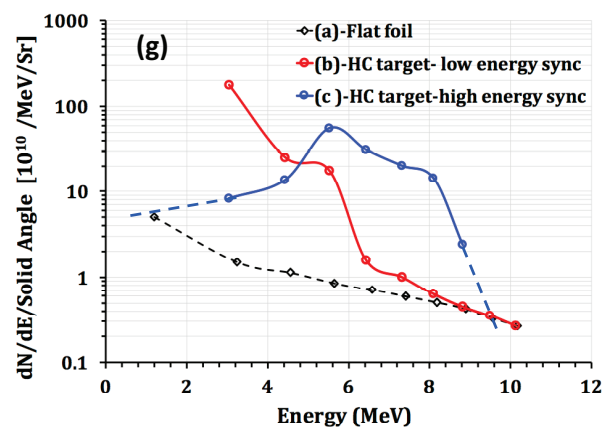

Figure 2. (a), (c) and (e) show the schematics of the experimental set-up used for the shots studying chromatic focussing and its tunability by varying coil pitch. The laser irradiated foil in all cases were 10 $\mu \mathrm{m} \mathrm{Au}$ foil and the coils in (c) and (e) had $\sim 450 \mu \mathrm{m}$ internal diameter and $\sim 3.3 \mathrm{~mm}$ length, but of different pitches to synchronise the lower and higher ends of the proton spectrum respectively. (b), (d) and (f) show the beam profiles obtained from the cases (a), (c) and (e) respectively. The dashed red circles show the exit circumference of the coil targets projected on the RCF plane. (g) shows the comparison between on-axis proton spectra obtained from the flat foil and the two HC targets shown in (b), (d) and (f), illustrating the tunable chromatic focussing by the HC target scheme. 


\section{Optimisation of TNSA proton beams using helical coil targets}

\subsection{Chromatic focusing and tunability}

By employing helical coils synchronised for different proton energies at the TARANIS laser system, focusing of multi-MeV protons has been achieved, as shown in figure 2. The coils used for the shots shown in figure 2 (c) and (e) were of same diameter and length, but had slightly different pitch to synchronise the lower and higher ends of the proton spectrum respectively [20]. Compared to a divergent proton beam typically obtained from flat foil targets, as shown in figure $2 b$, figure 2(d) show a strong focusing of $3 \mathrm{MeV}$ protons to a spot of approximately a mm diameter, which resulted in dose saturation of the RCF layer despite of the $70 \mathrm{~mm}$ distance between the target and the detector (the RCF distance for the flat foil shot shown in figure 2(b) was $23 \mathrm{~mm}$ ). The chromatic behaviour of the HC target can be clearly seen from the beam divergence of higher energy protons, which gradually increases with proton energy to the cone angle sustained by the $\mathrm{HC}$ (marked as a red dashed circle) at $9 \mathrm{MeV}$. A comparison between on-axis proton spectra for the flat foil and the HC coil is shown in figure. $2(\mathrm{~g})$, which shows two orders (and possibly higher due to the saturation of the RCF layer on axis for the $3 \mathrm{MeV}$ ) of magnitude increase in the proton flux at $\sim 3 \mathrm{MeV}$ compared to a flat foil shot. A collimated beam of few $\mathrm{MeV}$ protons would be highly relevant to many applications such as warm dense matter creation, neutron source development.

As discussed in section 2, the constant speed of the EM pulse allows tuning the energy range for beam optimisation by varying the coil's physical parameters, for instance by varying the coil pitch for a fixed diameter. As shown in the figure 2(f), increasing the pitch of the HC yielded stronger focussing of higher energy side of the proton spectrum, while the beam size for the lower energy protons (for ex. the $3 \mathrm{MeV}$, corresponding to the the 1st RCF layer shown in figure 2(f)) matches the projection of the coil aperture on the detector plane. The on-axis proton spectrum for this case is shown in figure $2(\mathrm{~g})$, which displays a significant increase in proton flux for the mid part of the spectrum, as the protons of this energy range remains in sync with the EM pulse for the most part of the coil length [20].

\subsection{Post-acceleration by helical coil targets}

The focusing of the proton beams by the HC targets shown above (figure 2(c) and (e)) were induced by the radial component of the electric field $\left(E_{r}\right)$ produced by the EM pulse in a helical geometry, as shown in the figure 1(b). The effect of the longitudinal component of the electric field $\left(E_{z}\right)$ in those cases were not pronounced due to their short coil length. Figure 3 highlights the salient features of the proton beam produced by a helical coil of $\sim 5.5 \mathrm{~mm}$ long (nearly double the length of the coils shown in figure 2$)$, with diameter and pitch $(\sim 700 \mu \mathrm{m}$ and $\sim 320 \mu \mathrm{m}$ respectively) chosen to synchronise a narrow slice of the proton spectrum around $7 \mathrm{MeV}$. As shown in the figure 3(c), 6-7 $\mathrm{MeV}$ protons co-propagate with the peak of the pulse for the entire duration they spend inside the coil, whereas, the higher and lower energy protons would remain in sync for a small fraction of their transit time. As expected, the lower energy protons (see figure 3(b)) are virtually unaffected by the EM pulse travelling along the coil.

As per the synchronisation graph shown in figure 3(c), one would expect a similar behaviour for $10 \mathrm{MeV}$ protons originating from the laser irradiated foil, as they would outrun the EM pulse electric field after travelling a few mm within the coil. However, as can be seen in figure 3(b), 

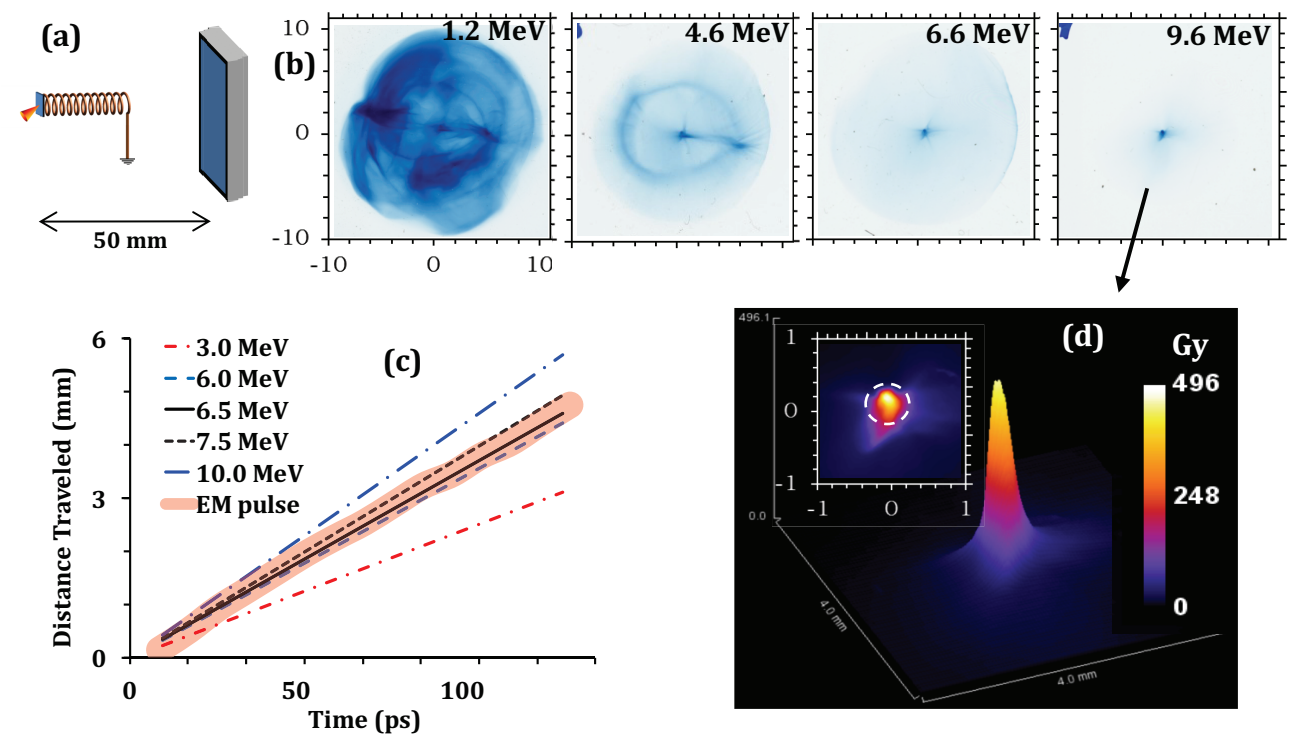

Figure 3. (a) shows the schematics of helical coil target and the experimental setup, (b) shows proton beam profiles obtained from the $5.5 \mathrm{~mm}$ long $\mathrm{HC}$ target of diameter and average pitch of $\sim 700 \mu \mathrm{m}$ and $\sim 320 \mu \mathrm{m}$ respectively. The spatial scale of the RCF images are represented by the angle sustained with respect to the proton sources at the rear side of the foil, located $50 \mathrm{~mm}$ in front of the detector. (c) The graph illustrate the synchronisation between the peak of the EM pulse travelling along the coil wire, projected on the coil axis (thick orange line), and the transit of different energy protons along the coil axis (thin lines as mentioned in the figure legend). (d) shows 3D dose profile of the the focussed spot on the last RCF layer, corresponding to a proton energy of $\sim 10 \mathrm{MeV}$. The insert shows a 2D dose profile compared to the internal diameter of the coil represented by the white dashed line.

the $\mathrm{HC}$ target produced a highly collimated beam of protons of energy $\sim 10 \mathrm{MeV}$, with nearly two orders of magnitude higher flux compared to the flat foil reference (the detector in this case was placed at $50 \mathrm{~mm}$ distance from the proton generating foil compared to $23 \mathrm{~mm}$ for the flat foil case in figure 2(a)). The on-axis proton spectrum obtained from this shot suggests that more than $10^{7}$ protons within a $\sim 10 \%$ bandwidth at $10 \mathrm{MeV}$ were contained within the focussed spot observed in the RCF layer. Furthermore, as shown in the insert of figure 3(d), the spot size of the $10 \mathrm{MeV}$ protons is less than the internal diameter of the coil, which suggest quasi-collimated propagation of the high energy protons after exiting the $\mathrm{HC}$ target. Since the $10 \mathrm{MeV}$ protons from the source would remain in sync with the EM pulse for only a small period of time, as shown in figure 3(c), one can assume that the focussed beam of $10 \mathrm{MeV}$ were produced by post-acceleration of the $\sim 7$ $\mathrm{MeV}$ protons along the coil, due to their prolonged exposure to the longitudinal component of the electric field produced by the EM pulse. This interpretation was broadly supported by particle tracing simulations [18] of the proton beam propagating through the helical coil.

\section{Conclusions}

We reported chromatic guiding and signature of post-acceleration of laser-driven protons by the HC targets at the 20TW TARANIS laser at QUB, UK. The chromatic nature of the HC target and its tunability was studied by varying the pitch of the coil, by which different sections of the proton 
spectra were efficiently guided to spots of sizes less than the internal diameter of the coil. Using the same approach with a longer coil, a quasi-collimated proton beam at $10 \mathrm{MeV}$ was produced which was estimated to contain more than $10^{7}$ particles within a $10 \%$ bandwidth. Since the long coil was designed to synchronise $\sim 7 \mathrm{MeV}$ protons, the observed pencil beam of $10 \mathrm{MeV}$ protons suggest a pronounced post-acceleration by a few $\mathrm{MeV}$ of the synchronised proton bunch guided by the coil. Since the amplitude of the EM pulse scales with the incident laser parameters [18], one would expect orders of magnitude increase in the electric field strength by employing Petawatt-class lasers systems, such as VULCAN PW at RAL, UK, which will lead to a significant boost in ion energy via post-accelerating the TNSA protons.

\section{Acknowledgments}

The authors acknowledge funding from EPSRC, (EP/J002550/1-Career Acceleration Fellowship held by S.K., EP/L002221/1, EP/K022415/1, EP/J500094/1 and EP/I029206/1), SBF-TR18 and GRK1203, EC-GA284464 and Invest Northern Ireland (POC-329).

\section{References}

[1] S.V. Bulanov and T.Z. Esirkepov, Phys. Lett. A, 299, 240, (2002); K. Ledingham and W. Galster, The British Journal of Radiology, 80, 855 (2007).

[2] M. Tabak et al., Phys. Plasmas, 1, 1626 (1994).

[3] M. Roth et al., Phys. Rev. Lett., 86, 436 (2001).

[4] P. Patel et al., Phys. Rev. Lett., 91, 125004 (2003).

[5] M. Koenig et. al, Plasma Phys. Control. Fusion, 47, B441 (2005).

[6] I. Spencer et al., Nucl. Instru. Methods B, 183, 449 (2001).

[7] S. Kar et. al, New J. Phys. 18, 053002 (2016).

[8] R.A. Snavely et al., Phys. Rev. Lett., 85, 2945 (2000).

[9] A. Macchi, M. Borghesi, and M. Passoni, Rev. Mod. Phys., 85, 751 (2013).

[10] S. Wilks et al., Phys Plasma, 8, 542 (2001).

[11] T. Toncian et al., Science, 312, 410 (2006).

[12] S. Kar et al., Phys. Rev. Lett., 100, 1, (2008).

[13] T. Bartal et al., Nat. Phys., 8, 139 (2011).

[14] M. Schollmeier et al., Phys. Rev. Lett., 101, 055004, (2008).

[15] K. Harres et al., Phys Plasma, 17, 023107 (2010).

[16] J. Fuchs et al., Nat. Phys. 2, 48 (2006); L. Robson et al., Nat. Phys. 3, 58 (2007).

[17] P. Antici et al., IEEE Trans. Plasma Sci. 36, 1843 (2008).

[18] S. Kar et al., Nat. Commun, 7, 10792 (2016).

[19] H. Ahmed et al., Nucl. Instru. Methods A, 829, 172 (2016).

[20] S. Kar et al., Phys Plasma, 23, 055711 (2016). 
[21] T. Dzelzainis et al., Laser Particle Beams, 28, 451 (2010).

[22] D Kirby et al., Laser Particle Beams, 29, 231 (2011). 